\title{
The Effects of ILeVO and VOTiVO on Root Penetration and Behavior of the Soybean Cyst Nematode, Heterodera glycines
}

Augustine Q. Beeman, Department of Plant Pathology and Microbiology, Iowa State University, Ames, IA 50011; Zach L. Njus and Santosh Pandey, Department of Electrical and Computer Engineering, Iowa State University, Ames, IA 50011; Gregory L. Tylka, ${ }^{\dagger}$ Department of Plant Pathology and Microbiology, Iowa State University, Ames, IA 50011

\begin{abstract}
The objective of this study was to determine the effects of ILeVO (fluopyram) and VOTiVO (Bacillus firmus I-1582) seed treatments on Heterodera glycines second-stage juvenile (J2) root penetration and behavior. In a growth chamber experiment, roots of soybeans grown from treated or untreated seeds were inoculated with $H$. glycines $\mathrm{J} 2 \mathrm{~s}$ at soil depths of 2.5 , 5 , or $7.5 \mathrm{~cm}$. ILeVO significantly reduced $H$. glycines root penetration compared with the untreated control, but only when J2s were inoculated at a soil depth of $2.5 \mathrm{~cm}$, which was near the seed. Changes in nematode behavior were assessed by collecting 60 -s videos of J2s after $2 \mathrm{~h}$ of exposure to exudates from treated seeds or radicles from treated seeds or from soil leachates in which treated seeds were planted. X- and ycoordinates of each of the 13 reference points were recorded every hour for $24 \mathrm{~h}$. A custom program analyzed and transformed the coordinates into nematode motion parameters (speed and total change in curvature). ILeVO, but not VOTiVO, seed exudates significantly reduced J2 speed

relative to the untreated control. Soil leachates from ILeVO or VOTiVO treatments had no consistent effect on $H$. glycines speed or total change in curvature compared with the untreated control. In another experiment, treated or untreated seeds were incubated in wells of 6-well tissue culture plates containing $11.5 \%$ Pluronic gel. Seeds were removed after $2 \mathrm{~h}$, and approximately $50 \mathrm{~J} 2 \mathrm{~s}$ then were pipetted into each well. The plates were scanned every $60 \mathrm{~min}$ for $24 \mathrm{~h}$, and the number of J2s in each well that moved a minimum distance of $\geq 300 \mu \mathrm{m}$ was determined using another custom software program. ILeVO, but not VOTiVO, significantly reduced the movement of $\mathbf{J} 2$ populations relative to control wells in which no seeds were added. And wells that had seeds, treated or not, yielded significantly less $\mathrm{J} 2$ movement compared with the no-seed control. The results of these experiments indicate that ILeVO reduces activity on $H$. glycines $\mathrm{J} 2 \mathrm{~s}$ but may not affect nematodes beyond a limited area surrounding the treated seed.
\end{abstract}

The soybean cyst nematode, Heterodera glycines Ichinohe, is the most damaging pathogen of soybean, Glycine max (L.) Merr., in the United States and Canada (Allen et al. 2017). Host resistance and growing nonhost crops in rotation with soybeans have been the principal $H$. glycines management strategies for decades (Niblack et al. 2006). However, both management options have limitations. H. glycines eggs can survive for a decade or more in soil without a host (Inagaki and Tsutsumi 1971); thus growing nonhost crops in rotations with soybean will not eliminate infestations. And $H$. glycines populations capable of reproducing on soybeans containing the most common source of resistance, PI 88788, have become more prevalent in recent years (McCarville et al. 2017; Mitchum et al. 2007; Niblack et al. 2008). These issues have stirred interest in alternative management strategies, including using nematode-protectant seed treatments.

Seed treatment is a method in which seeds are coated with formulated products containing active ingredients (AIs) designed to protect young plants against pests and diseases (Munkvold et al. 2014). Seed treatment options for soybeans include products with AIs that target fungi, oomycetes, insects, and nematodes. Although nematodeprotectant seed treatments have been studied since at least 1960 (O'Bannon and Reynolds 1960), they only have been available for soybeans since the mid-2000s. Both chemical nematicides and antagonistic microorganisms have been developed into commercially available seed treatments. There are several seed treatments for $H$.

${ }^{\dagger}$ Corresponding author: G. L. Tylka; E-mail: gltylka@iastate.edu

Funding: Bayer CropScience provided treated seed and funding for this research. The Iowa State University Graduate Minority Assistantship Program provided additional funding.

Accepted for publication 9 August 2018.

C 2019 The American Phytopathological Society glycines control on the market, and a number of new products are expected in the near future.

Seed treatments are a promising management tool for $H$. glycines and other plant-parasitic nematodes because the products deliver AIs directly to the seed and root zones, reducing the total amount of AI used per hectare relative to other soil application methods (Munkvold et al. 2014). However, the performance of nematode-protectant seed treatments, as measured by increased crop yields or reduced nematode reproduction, has been inconsistent in field trials (Bissonnette et al. 2018; Gaspar et al. 2014; Wheeler et al. 2013). One possible explanation for observed performance variability is interactions of the seed treatments with the environment (Wheeler et al. 2013).

VOTiVO (AI Bacillus firmus I-1582; BASF) and ILeVO (AI fluopyram; BASF) are two seed treatment products that contain AIs with documented effects on plant-parasitic nematodes. B. firmus I-1582 stimulates plant defenses and inhibits $H$. glycines hatching and motility (Schrimsher 2013). Fluopyram is both fungicidal (Avenot and Michailides 2010) and nematistatic (Faske and Hurd 2015) and affects both $H$. glycines and the causal pathogen of soybean sudden death syndrome, Fusarium virguliforme (Kandel et al. 2016; Zaworski 2014). Our understanding of the effects of both AIs on H. glycines as formulated seed treatments is limited. For example, it is unclear how VOTiVO and ILeVO affect nematodes in different parts of the soil profile or if the AIs move reliably on or through the roots in quantities sufficient for nematode control in different sections of the root.

The objectives of this research were to examine i) root protection conferred by ILeVO and VOTiVO at different soil depths, ii) the effects of exudates (seed and radicle) from treated seeds and soil leachates from soil in which treated seeds were planted on $H$. glycines motion, and iii) the effects of treated seeds on movement of $\mathrm{J} 2$ populations.

\section{Materials and Methods}

Seed treatments and source of $\boldsymbol{H}$. glycines inoculum. Seeds of an $H$. glycines-susceptible soybean cultivar (cv. Williams 82) were 
treated by Bayer CropScience personnel (Research Triangle Park, $\mathrm{NC}$ ) at commercial rates of AI: $0.15 \mathrm{mg}$ fluopyram/seed for ILeVO and $5 \times 10^{6} \mathrm{~B}$. firmus spores/seed for VOTiVO. No other fungicides or insecticides were applied to the seeds used in the experiments. The population of $H$. glycines used in this study was from field soil (Fruitfield coarse sand) collected in Muscatine, Iowa. The $H$. glycines population was determined to be HG type 2.5.7 (Niblack et al. 2002). The nematodes were cultured on $H$. glycines-susceptible soybean (cv. Williams 82) under greenhouse conditions. Females and cysts (dead females) were collected from 4- to 8-week-old $H$. glycines-infected soybeans by dislodging the nematodes on the surface of the roots with a stream of water and collecting the nematodes and any soil and root debris on a $250-\mu \mathrm{m}$-pore sieve. Females and cysts then were crushed with a motorized rubber stopper to release the eggs (Faghihi and Ferris 2000), and the H. glycines eggs were separated from the debris using sucrose centrifugation (Jenkins 1964). Microsieves (30- $\mu \mathrm{m}$ pore) were constructed from nylon mesh (Elko Filtering Co., Switzerland) and test tube caps (Wong et al. 1993). Eggs were pipetted on the microsieves and incubated in a dish of sterile distilled water at $25^{\circ} \mathrm{C}$. Second-stage juveniles (J2s) that hatched and moved through the sieves and into the dishes within 3 days were used for all experiments. Prior to experimental setup, the J2s were pipetted onto clean microsieves in dishes of sterile distilled water, and only the nematodes that moved through the sieves and into the dish within a few hours were used for experiments.

Root penetration experiment. The conical bottoms of 50-ml centrifuge tubes (Thermo Fisher Scientific Inc., Waltham, MA) were removed with a saw. Three holes (approximately $2 \mathrm{~mm}$ in diameter) were drilled in a straight line down the length of the tubes with a power drill. The capped tubes were inverted and filled with a pasteurized 2:1 sand:soil mixture ( $\mathrm{pH} 7.6$, organic matter 3.9\%). The experiment was organized as a two-factor factorial with three levels of seed treatment (ILeVO, VOTiVO, and untreated) and three inoculation depths (2.5, 5 , and $7.5 \mathrm{~cm}$ below the soil surface). Treated (ILeVO and VOTiVO) and untreated soybean seeds were planted approximately $2.5 \mathrm{~cm}$ deep, one seed per tube. The tubes were arranged in a growth chamber $\left(25^{\circ} \mathrm{C}, 16\right.$-h photoperiod) in a randomized complete block design with four replications per experimental run. The soybeans were watered with tap water daily for 5 days before $H$. $g l y$ cines inoculation. Roots of soybeans were inoculated with approximately $300 \mathrm{~J} 2 \mathrm{~s}$ at depths of $2.5,5$, and $7.5 \mathrm{~cm}$ below the soil line by pipetting nematodes in one of the holes drilled down the length of the tube. Two days after inoculation, the J2s that had penetrated the soybean roots were extracted. First, the roots were cut into 1to 2-cm-long pieces and homogenized using a Farberware kitchen blender (Meyer Corp., Vallejo, CA) for approximately $30 \mathrm{~s}$ (de Boer et al. 1999). The homogenate then was poured over a $250-\mu \mathrm{m}$-pore sieve on top of a $25-\mu \mathrm{m}$-pore sieve, and a motorized rubber stopper was used to grind root fragments on the top sieve (Faghihi and Ferris 2000). The J2s were collected on the $25-\mu \mathrm{m}$-pore sieve and quantified visually using a Nikon AZ100 microscope (Nikon Instruments Inc., Tokyo, Japan) at $20 \times$ magnification. The experiment was repeated once.

Seed and radicle exudate collection. Seed and radicle exudates were collected using the method reported by Beeman and Tylka

Table 1. Total number of Heterodera glycines juveniles (J2s) analyzed for exudate (seed and radicle) and soil leachate experiments over two experimental runs

\begin{tabular}{lccc}
\hline & \multicolumn{3}{c}{ Total number of J2s analyzed } \\
\cline { 2 - 4 } Treatment & Seed exudates & Radicle exudates & Soil leachates \\
\hline ILeVO & 15 & 10 & 10 \\
VOTiVO & 8 & 9 & 14 \\
Untreated & 12 & 12 & 12 \\
\hline Controls & Seed and radicle exudate experiments & Soil leachates \\
\hline Heat killed & \multicolumn{2}{|c}{13} \\
Water & 8 & 9 \\
Blank & \multicolumn{2}{c}{ N/A } \\
\hline
\end{tabular}

(2018). Seeds were incubated in sterile distilled water at a ratio of one seed per $5 \mathrm{ml}$ water for $1 \mathrm{~h}$ on a platform shaker $(100 \mathrm{rpm})$. Radicle exudates were collected similarly; first seeds were germinated on $1.5 \%$ water agar, and radicles (5 to $7 \mathrm{~cm}$ length) of intact, 3 -day-old soybean seedlings were incubated on a platform shaker at $100 \mathrm{rpm}$ for $1 \mathrm{~h}$ in sterile distilled water at a ratio of one radicle per $5 \mathrm{ml}$ water. During radicle exudate collection, the shoots of the seedlings (including the seed coat) were suspended on cheesecloth and were not in contact with the sterile water. All exudates were poured over 30$\mu \mathrm{m}$-pore nylon mesh to remove debris and stored at approximately $4^{\circ} \mathrm{C}$ before experimental setup.

Soil leachate collection. Microsieves $(30-\mu \mathrm{m}$ pore, $2 \mathrm{~cm}$ height, and $2 \mathrm{~cm}$ diameter) were filled with the pasteurized, $2: 1$ sand:soil mixture described previously and watered with sterile distilled water to approximate field capacity $(2.5 \mathrm{ml}$ per microsieve). Treated (VOTiVO and ILeVO) and untreated Williams 82 seeds were planted in the microsieves at a ratio of one seed per microsieve and incubated at $25^{\circ} \mathrm{C}$ for $24 \mathrm{~h}$. Another $2.5 \mathrm{ml}$ of sterile distilled water then was added to each microsieve, and the leachates escaping from the bottom of each microsieve were collected in wells of 6-well tissue culture plates (Corning Inc., Corning, NY). Solutions collected from microsieves with no seeds, hereafter referred to as the blank, and sterile distilled water served as controls. Each soil leachate treatment had three replications that subsequently were pooled. The leachates then were centrifuged at $2,200 \times g$ for $2 \mathrm{~min}$ to remove soil debris, and the supernatant was stored at approximately $4^{\circ} \mathrm{C}$ before experimental setup.

Effect of exudates and leachates on $\mathrm{H}$. glycines motion. Changes in speed and curvature of individual $H$. glycines $\mathrm{J} 2 \mathrm{~s}$ were studied using a method developed by Jensen et al. (2018). Exudates (seed and radicle) and soil leachates were tested in separate experiments. $H$. glycines $\mathrm{J} 2 \mathrm{~s}$ were pipetted into $1.5-\mathrm{ml}$ centrifuge tubes containing exudates or leachates for $2 \mathrm{~h}\left(25^{\circ} \mathrm{C}\right)$. The $\mathrm{J} 2 \mathrm{~s}$ then were rinsed three times with sterile distilled water and then suspended in water; 10 to $15 \mu \mathrm{l}$ of the suspension was pipetted onto glass slides with a 20-mm-diameter coverslip and imaged with a QICAM 12bit Color Fast 1394 camera (Ziosoft Inc., Tokyo, Japan) connected to a Leica M205-C microscope (Leica Microsystems Inc., Buffalo Grove, IL) at $63 \times$ magnification $(6.3 \times$ zoom, $10 \times$ lens, $1 \times$ camera $)$ with overhead lighting. Each nematode was imaged for 1 min with a rate of 10 frames per second. In both experiments, water served as a positive control and heat-killed nematodes as a negative control. The heat-killed nematodes were generated by placing live nematodes in sterile distilled water in a tube and immersing the tube in boiling water for $2 \mathrm{~min}$. The videos were processed using two custom software programs written in MATLAB (MathWorks Inc., Natick,

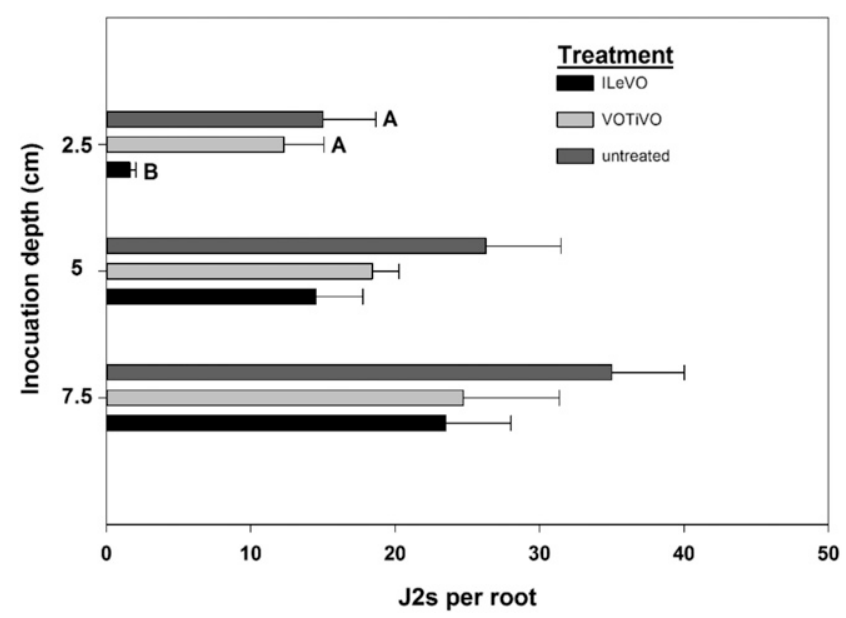

Fig. 1. Number of Heterodera glycines second-stage juveniles (J2s) recovered from roots of soybeans grown from treated seeds after inoculation with approximately $300 \mathrm{~J} 2 \mathrm{~s}$ at three different soil depths in a growth chamber experiment. Error bars are standard errors of the means. Different letters to the right of the bars in the same inoculation depth indicate significant differences according to Tukey's honest significant difference test $(\alpha=0.05)$. 
MA) to measure changes in nematode behavior (Jensen et al. 2018). Briefly, the software assigned 13 tracking points along the length of a nematode body and recorded the $\mathrm{x}$ - and $\mathrm{y}$-coordinates of each point. The $H$. glycines motion parameters that were calculated from the $\mathrm{x}$ - and $\mathrm{y}$-coordinates were speed (13 discrete body points) and total change in curvature (whole body). Average speed of each body point over the 1-min video was determined by averaging the instantaneous speeds, in $\mu \mathrm{m} / \mathrm{s}$, of the body points at each of the frames over the 60-s video (Jensen et al. 2018; Njus et al. 2015). The speed of the anterior-most body point, referred hereafter as the head point, was analyzed for each nematode and considered the representative speed for the whole body. Curvature was assessed by calculating the radius of a hypothetical circle tangent to two lines connecting three continuous tracking points on a nematode body (Jensen et al. 2018; Likitlersuang et al. 2012), and values were expressed in $1 / \mu \mathrm{m}$. Total change in curvature was calculated by summing the instantaneous change in curvature at each of the body points in successive frames and averaging the value over the length of the video. Eight to 15 nematode juveniles were analyzed per treatment over two experimental runs (Table 1).

Effect of seed treatments on movement of $\mathrm{H}$. glycines populations. Movement of populations of $H$. glycines $\mathrm{J} 2 \mathrm{~s}$ after exposure to seed treatments was measured using a scanner assay described by Jensen et al. (2018). Wells of 24-well tissue culture plates (Corning Inc., Corning, NY) were filled with $500 \mu \mathrm{l}$ of $11.5 \%$ Pluronic F-127 gel (Sigma Life Science, St. Louis, MO). A single treated (ILeVO or VOTiVO) or untreated Williams 82 seed was inserted into each well. Wells without seeds, referred to hereafter
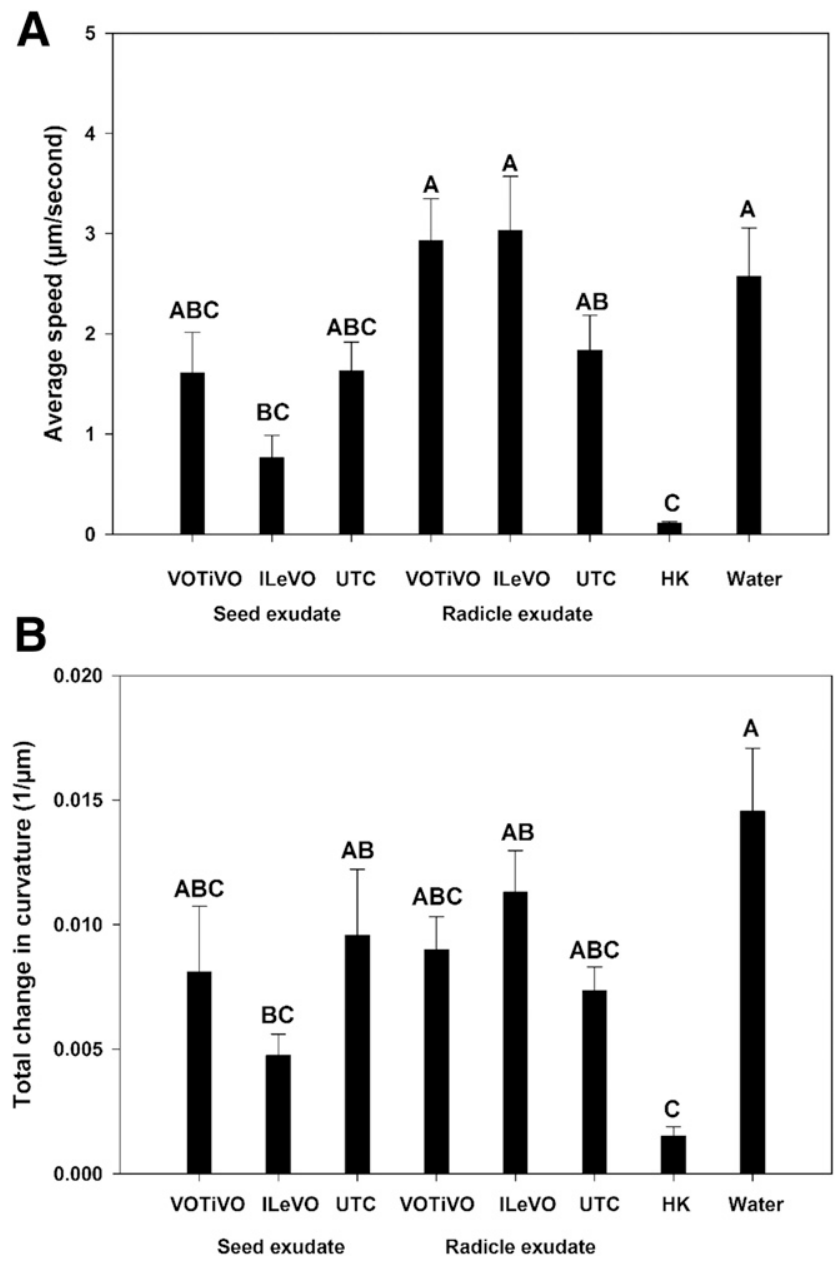

Fig. 2. Mean ( \pm standard error of the mean) head speed $(\mathbf{A})$ and total change in curvature (B) of Heterodera glycines second-stage juveniles (J2s) exposed to seed and radicle exudates for $2 \mathrm{~h}$. HK = heat-killed J2s, UTC = untreated control. Different letters indicate significant differences according to Tukey's honest significant difference test $(\alpha=0.05)$. as the blank, served as a control. Seeds were incubated in the wells at $25^{\circ} \mathrm{C}$ for $2 \mathrm{~h}$ before being removed with clean forceps. Then, $54 \pm 14$ $\mathrm{J} 2 \mathrm{~s}$ (mean \pm standard deviation) were pipetted into each well. The 24-well plate was incubated on an EPSON Perfection V750-M Pro scanner (Seiko Epson Corp., Japan) housed inside a temperaturecontrolled poly(methyl methacrylate) box $\left(25^{\circ} \mathrm{C}\right)$. The plate was scanned (2,400 dpi) automatically every hour for $24 \mathrm{~h}$ (Jensen et al. 2018). After image collection, the J2s in each image were marked with a cursor in a custom program written in MATLAB, and the x- and ycoordinates of the centroids of the $\mathrm{J} 2 \mathrm{~s}$ in each of the wells at every time point was determined by the program. The $\mathrm{x}$ - and $\mathrm{y}$-coordinates of all $\mathrm{J} 2 \mathrm{~s}$ were analyzed in another custom MATLAB program to calculate the percent of the $H$. glycines population that had moved $300 \mu \mathrm{m}$ or more during the hour between when each pair of images was captured (Jensen et al. 2018). Percent movement data were collected for each time point in the experiment. There were three to four replications per run, and the experiment was repeated once.

Statistical analyses. The data from all experiments were analyzed in SAS Version 9.4 (SAS Institute, Cary, NC). The combined data from each experiment were subjected to analysis of variance (ANOVA) using the normal distribution, and residual plots were examined before additional analysis. Main effects (seed treatment and inoculation depth) and interactive effects were analyzed for significance in the root penetration experiment using PROC GLIMMIX. For the scanner experiment, repeated measures analysis was conducted using PROC MIXED. Results from the scanner experiment were then averaged for $0-6 \mathrm{~h}, 7-16 \mathrm{~h}$, and $17-24 \mathrm{~h}$, and treatments were compared for each of these time periods. For all experiments, least-squared means were separated using Tukey's honest significant difference (HSD) test (PROC GLIMMIX, $\alpha=0.05$ ).

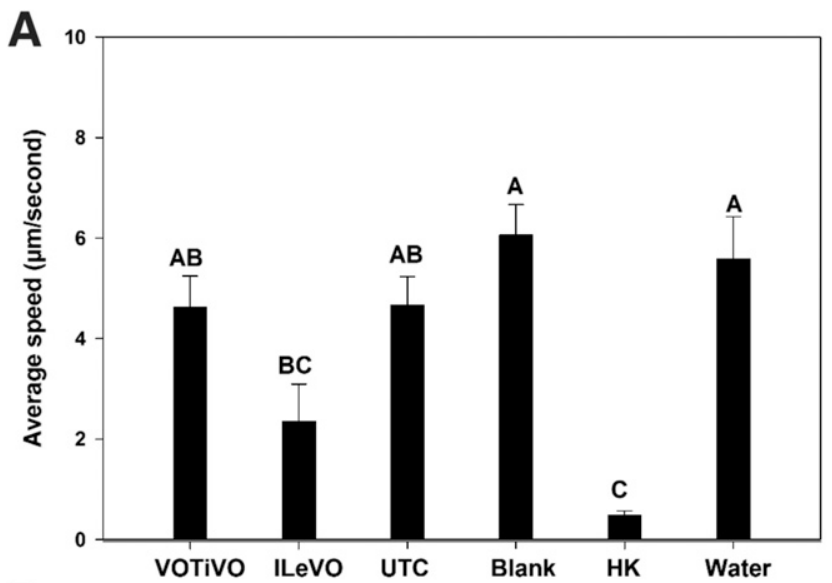

B

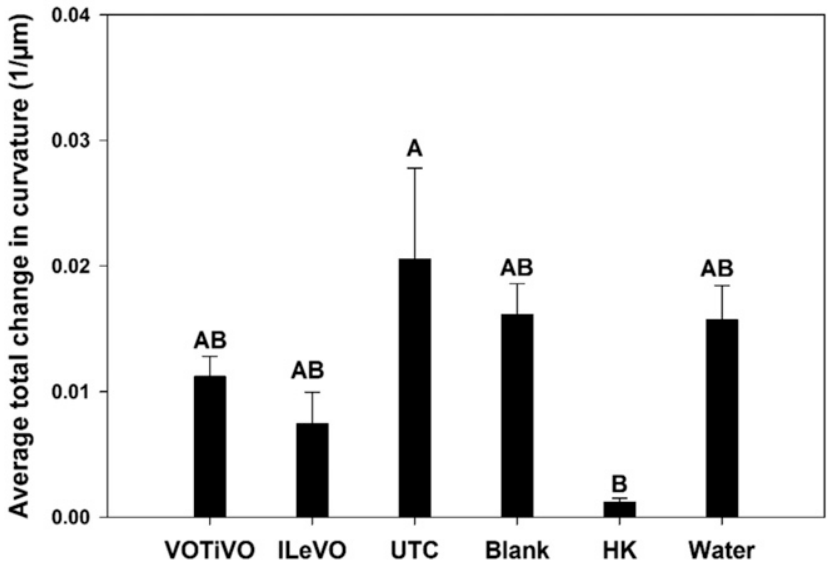

Fig. 3. Mean ( \pm standard error of the mean) head speed $(\mathbf{A})$ and total change in curvature (B) of Heterodera glycines second-stage juveniles (J2s) exposed to soil leachates for $2 \mathrm{~h}$. HK = heat-killed J2s, UTC = untreated control. Different letters indicate significant differences according to Tukey's honest significant difference test $(\alpha=0.05)$. 


\section{Results}

Root penetration experiment. When analyzed as a two-factor factorial experiment, significant main effects were detected for seed treatment and inoculation depth. There was no interaction between the main factors. Data then were analyzed by seed treatment as a single factor experiment. Across all inoculation depths combined, ILeVO significantly reduced root penetration relative to the untreated control (data not shown). However, when the data were analyzed for effects of seed treatment at different inoculation depths, there was a significant, $87 \%$ reduction in the number of $\mathrm{J} 2 \mathrm{~s}$ that had penetrated roots at the $2.5-\mathrm{cm}$ inoculation depth compared with the untreated control (Fig. 1). There were no significant effects of ILeVO at the 5- or 7.5-cm inoculation depths (Fig. 1). VOTiVO did not affect root penetration at any inoculation depth (Fig. 1) in our experiments.

Effect of exudates and leachates on $\mathrm{H}$. glycines motion. For each experiment, the number of $\mathrm{J} 2 \mathrm{~s}$ analyzed in each treatment is listed in Table 1. The experiments studying the effects of seed and radicle exudates were conducted at different times, and the data were analyzed separately (Figs. 2 and 3). For seed exudates, motion of the heads of nematodes incubated in the ILeVO treatment was numerically lower than in the VOTiVO and untreated seed exudates, but not significantly different. ILeVO and VOTiVO radicle exudates did not affect the head speed of $\mathrm{J} 2$ compared with that of untreated radicle exudates (Fig. 2A). The speed of J2s exposed to ILeVO seed exudate was significantly less than that of $\mathrm{J} 2 \mathrm{~s}$ exposed to the water control and VOTiVO and ILeVO radicle exudates (Fig. 2A). ILeVO or VOTiVO seed and radicle exudates did not significantly affect average total change in curvature of the nematodes relative to the untreated seed or radicle exudate control (Fig. 2B). However, J2s exposed to ILeVO seed exudates had significantly less total change in curvature relative to the water control.

In the soil leachate experiment, there were no significant differences in the speed of head motion (Fig. 3A) or change in curvature (Fig. 3B) among the seed treatments and the untreated control. However, ILeVO soil leachates significantly reduced the speed of motion of the head of $\mathrm{H}$. glycines J2s relative to the blank control (Fig. 3A).

Limited levels of motion and change in curvature were detected in the heat-killed control nematodes in both experiments. This motion was due to small vibrations or drift of the dead nematodes (Figs. 2 and 3).

Effect of seed treatments on movement of $H$. glycines populations. In general, populations of $H$. glycines in the treatments had similar levels of movement in the first few hours, but amounts of movement diverged according to treatment at later time points (Fig. 4A). The movement of the nematode populations in the ILeVO treatment was less than in the untreated control at most of the time points (Fig. 4A). The results of the repeated measures analysis revealed significant interactive effects of treatment and time $(P<0.0001)$. Consequently, average percent movement over three time periods $(0-6 \mathrm{~h}, 7-16 \mathrm{~h}, 17-24 \mathrm{~h})$ was calculated and compared. From 0 to $6 \mathrm{~h}$, movement of the $H$. glycines populations was similar across all treatments, with the exception of a significant difference between the blank and ILeVO treatments (Fig. 4B). Between 7 and $16 \mathrm{~h}$, nematodes in the ILeVO treatment moved at a significantly lower rate compared with the untreated, the VOTiVO, and the blank control treatments (Fig. 4C). From 17 to 24 h, movement across all treatments was reduced to less than $25 \%$, with the blank treatment having significantly greater movement than the other three
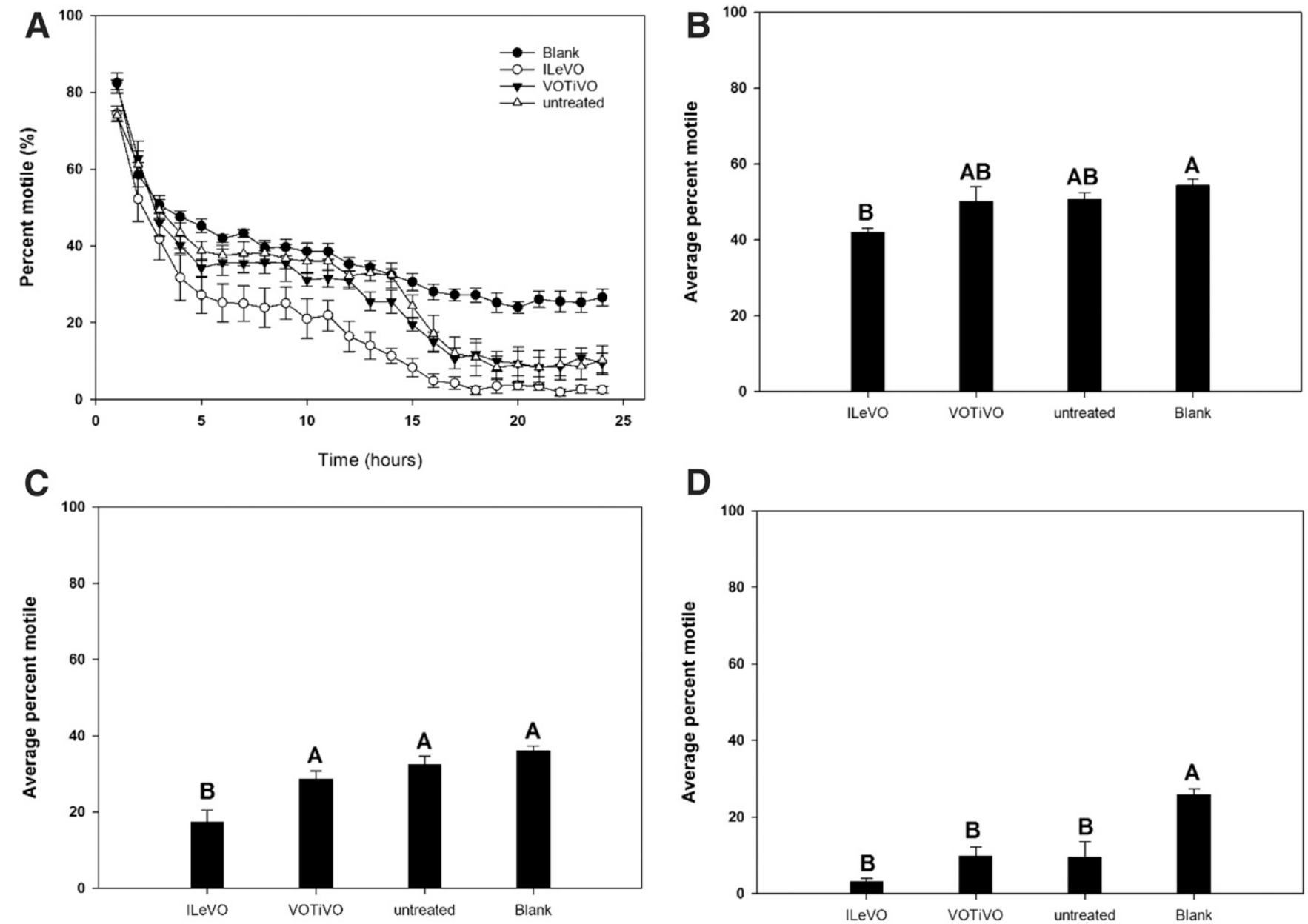

Fig. 4. Movement of Heterodera glycines second-stage juveniles (J2s) in 3-cm wells that previously contained treated or untreated seeds. (A) Average percent movement (mean \pm standard error of the mean) at each $1 \mathrm{~h}$ time point, (B) movement of $H$. glycines $\mathrm{J} 2$ populations averaged from 0 to $6 \mathrm{~h}$, (C) movement of $H$. glycines $\mathrm{J} 2 \mathrm{~s}$ from 7 to $16 \mathrm{~h}$, (D) movement of $H$. glycines J2s from 17 to $24 \mathrm{~h}$. Error bars indicate standard errors of the mean. Letters above bars in B-D indicate significant differences according to Tukey's honest significant difference test $(\alpha=0.05)$ 
treatments that contained a seed prior to introduction of the nematode (Fig. 4D).

\section{Discussion}

The seed treatment ILeVO affected most of the H. glycines parameters studied in our experiments. The effects were evident when the $\mathrm{J} 2 \mathrm{~s}$ were in close proximity to ILeVO-treated seeds or in ILeVO seed exudates but not when the nematodes were exposed to ILeVO radicle exudates or leachates from soil containing ILeVO-treated seeds. In contrast, VOTiVO had no effect on the $H$. glycines parameters we measured in our experiments.

ILeVO conferred protection to soybean roots when $H$. glycines $\mathrm{J} 2 \mathrm{~s}$ were inoculated at a $2.5 \mathrm{~cm}$ soil depth near the planted seeds. However, root protection from ILeVO was not observed at 5 and $7.5 \mathrm{~cm}$ nematode inoculation depths, which was 2.5 and $5 \mathrm{~cm}$ below the seed. This result may indicate that ILeVO protects soybean roots against $H$. glycines infection in a limited area near the treated seed. Similar results were reported for roots of cotton plants grown from abamectin-treated seeds, where initial Meloidogyne incognita galls occurred further down the cotton taproot grown from abamectintreated seed compared with untreated check plants (Faske and Starr 2007). Using an assay described by Jensen et al. (2018), we also found exudates from ILeVO-treated seeds significantly reduced the speed of motion of the $H$. glycines head and curvature of the body, relative to a water-only control. But ILeVO and VOTiVO seed and radicle exudates and leachates from soil in which treated seeds were planted had no consistent effect on the motion of the head of $H$. glycines $\mathrm{J} 2 \mathrm{~s}$ relative to that of $\mathrm{J} 2$ in the untreated seed controls. The lack of effects of radicle exudates and soil leachates from seeds treated with B. firmus I-1582 (AI of VOTiVO) and fluopyram (AI of ILeVO) on the nematode J2s may indicate that the seed treatment AIs did not travel in large quantities on roots or in the soil in our experiments. However, it is possible that both seed treatments could have greater mobility in soils with different properties ( $\mathrm{pH}$, organic matter, etc.) or under different watering regimes. We did not test leachates from different soil types to assess this hypothesis.

The scanner assay, also described in Jensen et al. (2018), estimated the percentage of $H$. glycines $\mathrm{J} 2 \mathrm{~s}$ in a population that traveled a critical threshold distance $(\geq 300 \mu \mathrm{m})$ over $1 \mathrm{~h}$ in wells that contained treated or untreated seeds for $2 \mathrm{~h}$ prior to the addition of the nematodes. Regardless of treatment, movement of the $\mathrm{J} 2$ populations decreased over time. This decreased movement trend of the J2s was possibly due to lack of external chemical stimuli or the gradual depletion of oxygen in the wells over time. ILeVO-treated seeds significantly reduced the movement of $H$. glycines populations between 7 and $16 \mathrm{~h}$ after the start of the experiment, relative to an untreated seed control (Fig. 4C). This result indicates that the amount of fluopyram that moved from the treated seed into the Pluronic gel after $2 \mathrm{~h}$ was enough to affect $H$. glycines movement. Thus, it is possible that an ILeVO-treated seed would leach fluopyram into the soil surrounding the seed zone and inhibit movement of nearby $\mathrm{J} 2 \mathrm{~s}$, potentially resulting in reduced nematode infection and plant damage.

VOTiVO did not affect any aspect of $H$. glycines biology that was measured in our experiments. Schrimsher (2013) reported that $H$. glycines $\mathbf{J} 2 \mathrm{~s}$ were immobilized when incubated with $B$. firmus concentrations ranging from $1 \times 10^{6}$ to $1 \times 10^{7} \mathrm{cfu} / \mathrm{ml}$. However, in another study, $B$. firmus did not immobilize $\mathrm{J} 2 \mathrm{~s}$ or reduce nematode root penetration (Beeman and Tylka 2018). There are a few possible explanations for the lack of activity of VOTiVO on $\mathrm{H}$. glycines in our study. The seeds were treated at a commercial rate of $5 \times 10^{6}$ spores/ seed, and it is conceivable that the number of spores coming off the seed and into the soil would be less than the effective concentrations reported by Schrimsher (2013). Additionally, the spores of the bacteria likely need to germinate and reproduce to be effective, and the duration of our experiments may have been too short for $B$. firmus to reach densities necessary for $H$. glycines control. VOTiVO is reported to induce plant defenses (Schrimsher 2013), potentially leading to increased plant biomass relative to an untreated control. We did not measure any plant growth parameters in our experiments. Lastly, the $B$. firmus I-1582 used in our experiments may not have been viable, something that we did not check. Repeating the same studies with seeds treated with new and different batches of $B$. firmus I-1582 would be necessary to explore this possibility. Additional research with altered experimental conditions and measured plant growth variables is necessary to fully understand the nematodeprotectant capabilities of VOTiVO.

There are a few challenges in measuring behavioral differences in plant-parasitic nematodes compared with other soil-dwelling nematodes. In general, plant-parasitic nematodes are less active than other nematodes inhabiting the soil, such as Caenorhabditis elegans. For example, in our experiments, $H$. glycines $\mathrm{J} 2 \mathrm{~s}$ in control treatments had average head motion speeds of approximately $2-6 \mu \mathrm{m} / \mathrm{s}$, whereas centroid points on the body of $C$. elegans have been reported to move at speeds around $100 \mu \mathrm{m} / \mathrm{s}$ or more when measured with the same or similar software and image-capturing techniques (Njus et al. 2015; Saldanha et al. 2013). Also, the movement of the nematode populations in the scanner was below $50 \%$ for most of the experiment and below $15 \%$ by the end of the experiment for the treatments that had a seed in the well prior to placement of the nematodes in the wells. Because $H$. glycines activity under ambient conditions started at a relatively low baseline in our studies, it may have been more difficult to discern treatment effects than it would be using more active nematodes such as $C$. elegans. It is also unclear whether other, unmeasured factors in the studies had an impact on the baseline activity of the nematodes that further challenged comparing treatments. ILeVO reduced root penetration and movement of $\mathrm{J} 2 \mathrm{~s}$ in some experiments, but it is unclear whether such a reduction would result in reduced nematode reproduction under less controlled conditions. It is important to note that any effects observed in vitro may not be relevant in the field environment (Spence et al. 2008). Similarly, lack of effects in our laboratory studies may not mean that such effects do not occur in the field. In our experiments, we attempted to study the effects of these seed treatments on specific aspects of $H$. glycines biology under controlled experimental conditions. Additional work examining the fitness of nematodes treated with sublethal amounts of ILeVO and field efficacy trials are necessary to determine whether behavioral effects of the compound on $H$. glycines result in meaningful control in the context of the field.

Our results suggest that ILeVO has activity against $H$. glycines, but its effects on the nematode may dissipate as the roots grow away from the treated seed. However, significant plant biomass increases have been reported by delaying nematode inoculation by several days (Huang and Ploeg 2001). Brief periods of soybean root protection conferred by ILeVO (and possibly VOTiVO) therefore could result in higher soybean biomass accumulation and yields. Field studies to understand the interactions of seed treatments with environmental factors and nematode densities, as well as economic cost-benefit analyses of the different seed treatment options, could enhance the utility of this management strategy.

\section{Acknowledgments}

We thank Chelsea Harbach for assistance in statistical analysis.

\section{Literature Cited}

Allen, T. W., Bradley, C. A., Sisson, A. J., Byamukama, E., Chilvers, M. I., Coker C. M., Collins, A. A., Damicone, J. P., Dorrance, A. E., Dufault, N. S., Esker, P. D., Faske, T. R., Giesler, L. J., Grybauskas, A. P., Hershman, D. E., Hollier, C. A., Isakeit, T., Jardine, D. J., Kelly, H. M., Kemerait, R. C., Kleczewski, N. M., Koenning, S. R., Kurle, J. E., Malvick, D. K., Markell, S. G., Mehl, H. L., Mueller, D. S., Mueller, J. D., Mulrooney, R. P., Nelson, B. D., Newman, M. A., Osborne, L., Overstreet, C., Padgett, G. B., Phipps, P. M., Price, P. P., Sikora, E. J., Smith, D. L., Spurlock, T. N., Tande, C. A., Tenuta, A. U., Wise, K. A., and Wrather, J. A. 2017. Soybean yield loss estimates due to diseases in the United States and Ontario, Canada from 2010 to 2014. Plant Health Prog. 18:19-27.

Avenot, H. F., and Michailides, T. J. 2010. Progress in understanding molecular mechanisms and evolution of resistance to succinate dehydrogenase inhibiting (SDHI) fungicides in phytopathogenic fungi. Crop Prot. 29:643-651.

Beeman, A. Q., and Tylka, G. L. 2018. Assessing the effects of ILeVO and VOTiVO seed treatments on hatching, motility, root penetration, and reproduction of the soybean cyst nematode. Phytopathology 102:107-113.

Bissonnette, K. M., Marett, C. C., Mullaney, M. P., Gebhart, G. D., Kyveryga, P., Mueller, T. A., and Tylka, G. L. 2018. Effects of Clariva Complete Beans seed 
treatment on Heterodera glycines reproduction and soybean yield in Iowa. Plant Health Prog. 19:1-8.

de Boer, J. M., Yan, Y., Wang, X., Smant, G., Hussey, R. S., Davis, E. L., and Baum, T. J. 1999. Developmental expression of secretory $\beta-1,4-$ endoglucanases in the subventral esophageal glands of Heterodera glycines. Mol. Plant-Microbe Interact. 12:663-669.

Faghihi, J., and Ferris, J. M. 2000. An efficient new device to release eggs from Heterodera glycines. J. Nematol. 32:411-413.

Faske, T. R., and Hurd, K. 2015. Sensitivity of Meloidogyne incognita and Rotylenchulus reniformis to fluopyram. J. Nematol. 47:316-321.

Faske, T. R., and Starr, J. L. 2007. Cotton root protection from plant-parasitic nematodes by abamectin-treated seed. J. Nematol. 39:27-30.

Gaspar, A. P., Marburger, D. A., Mourtzinis, S., and Conley, S. P. 2014. Soybean seed yield response to multiple seed treatment components across diverse environments. Agron. J. 106:1955-1962.

Huang, X., and Ploeg, A. T. 2001. Effect of plant age and Longidorus africanus on the growth of lettuce and carrot. J. Nematol. 33:137-141.

Inagaki, H., and Tsutsumi, M. 1971. Survival of the soybean cyst nematode, Heterodera glycines Ichinohe (Tylenchida:Heteroderidae) under certain storing conditions. Appl. Entomol. Zool. (Jpn.) 6:156-162.

Jenkins, W. R. 1964. A rapid centrifugal-flotation technique for separating nematodes from soil. Plant Dis. Rep. 48:692.

Jensen, J. P., Beeman, A. Q., Njus, Z., Kalwa, U., Pandey, S., and Tylka, G. L. 2018. Movement and motion of the soybean cyst nematode, Heterodera glycines, populations and individuals in response to abamectin. Phytopathology 108:885-891.

Kandel, Y. R., Wise, K. A., Bradley, C. A., Chilvers, M. I., Tenuta, A. U., and Mueller, D. S. 2016. Fungicide and cultivar effects on sudden death syndrome and yield of soybean. Plant Dis. 100:1339-1350.

Likitlersuang, J., Stephens, G., Palanski, K., and Ryu, W. S. 2012. C. elegans tracking and behavioral measurement. J. Vis. Exp. 69:e4094.

McCarville, M. C., Marett, C. C., Mullaney, M. P., Gebhart, G. D., and Tylka, G. L. 2017. Increase in soybean cyst nematode virulence and reproduction on resistant soybean varieties in Iowa from 2001 to 2015 and its effects on soybean yields. Plant Health Prog. 18:146-155.

Mitchum, M. G., Wrather, J. A., Heinz, R. D., Shannon, J. G., and Danekas, G. 2007. Variability in distribution and virulence phenotypes of Heterodera glycines in Missouri during 2005. Plant Dis. 91:1473-1476.
Munkvold, G. P., Watrin, C., Scheller, M., Zeun, R., and Olaya, G. 2014. Benefits of chemical seed treatments on crop yield and quality. Pages 89-103 in: Global Perspectives on the Health of Seeds and Plant Propagation Material. M. L. Gullino and G. P. Munkvold, eds. Springer, Netherlands.

Niblack, T. L., Arelli, P. R., Noel, G. R., Opperman, C. H., Orf, J. H., Schmitt, D. P., Shannon, J. G., and Tylka, G. L. 2002. A revised classification scheme for genetically diverse populations of Heterodera glycines. J. Nematol. 34: 279-288.

Niblack, T. L., Colgrove, A. L., Colgrove, K., and Bond, J. P. 2008. Shift in virulence of soybean cyst nematode is associated with use of resistance from PI 88788. Plant Health Prog. doi.org/10.1094/PHP-2008-0118-01-RS

Niblack, T. L., Lambert, K. N., and Tylka, G. L. 2006. A model plant pathogen from the Kingdom Animalia: Heterodera glycines, the soybean cyst nematode. Annu. Rev. Phytopathol. 44:283-303.

Njus, Z., Feldmann, D., Brien, R., Kong, T., Kalwa, U., and Pandey, S. 2015. Characterizing the effect of static magnetic fields on $C$. elegans using microfluidics. Adv. Biosci. Biotechnol. 6:583-591.

O'Bannon, J. H., and Reynolds, H. W. 1960. Preliminary studies with DBCP cotton seed treatment for controlling the root-knot nematode. Plant Dis. Rep. 44:484-486.

Saldanha, J. N., Parashar, A., Pandey, S., and Powell-Coffmann, J. A. 2013. Multiparameter behavioral analyses provide insights to mechanisms of cyanide resistance in Caenorhabditis elegans. Toxicol. Sci. 135:156-168.

Schrimsher, D. W. 2013. The studies of plant host resistance to the reniform nematode in upland cotton and the effects of Bacillus firmus GB-126 on plant-parasitic nematodes. M.S. thesis. Auburn University, Auburn, AL.

Spence, K. O., Lewis, E. E., and Perry, R. N. 2008. Host-finding and invasion by entomopathogenic and plant-parasitic nematodes: Evaluating the ability of laboratory bioassays to predict field results. J. Nematol. 40:93-98.

Wheeler, T. A., Lawrence, K. S., Porter, D. O., Keeling, W., and Mullinix, B. G. 2013. The relationship between environmental variables and response of cotton to nematicides. J. Nematol. 45:8-16.

Wong, A. T. S., Tylka, G. L., and Hartzler, R. G. 1993. Effects of eight herbicides on in vitro hatching of Heterodera glycines. J. Nematol. 25:578-584.

Zaworski, E. R. 2014. Effects of ILeVO ${ }^{\circledR}$ on soybean sudden death syndrome and soybean cyst nematode. Graduate Theses and Dissertations. Paper 14261. Iowa State University, Ames, IA. 